\title{
Macrofauna bentónica asociada a bancos ostrícolas en las lagunas costeras Carmen, Machona y Mecoacán, Tabasco, México
}

\author{
P.V. Susan Tepetlan \& D. Aldana Aranda \\ CINVESTAV Unidad Mérida, Antigua Carretera a Progreso Km. 6., A. P 73 Cordemex, C. P 97310, Mérida, Yucatán, \\ México; psusan@mda.cinvestav.mx; beatle12@hotmail.com, daldana@mda.cinvestav.mx.
}

Received 13-VII-2007. C Corrected 15-XI-2007. Accepted 13-II-2008.

\begin{abstract}
Benthic macrofauna associated to oyster banks in the coastal lagoons Carmen, Machona and Mecoacán, Mexico. Oyster beds have an important impact on coastal ecosystems. In this work, samplings were done during the dry, the rainy and the "North wind" seasons, in eight oyster reefs at the Lagoons of Mecoacán, Carmen and Machona, in Tabasco, Mexico. The $\alpha$ diversity was calculated with the Shannon-Wiener, Simpson and Margalef indexes. An Analysis of Variance was done to evaluate if there was a difference in the average of the indexes at each reef in every season, verifying the assumptions of normality and variance homogeneity. In the case of mollusk richness, we found 13 families, 13 genera and 15 species of gastropods, as well as 8 families, 10 genera and 11 species of bivalves. Seven families, 11 genera and 12 species of crustaceans were recorded. In the case of mollusks, the highest average value of species richness was recorded during the dry season, in Puente de Ostión reef $(\mathrm{DMg}=13.6)$, whereas in the case of species diversity, the highest average value was recorded during the "North wind" season, at Largo $\left(\mathrm{H}^{\prime}=0.77\right)$. The dominant mollusks during the three seasons were Ischadium recurvum, Isognomon alatus and Crepidula plana. In the case of crustaceans, the highest average value, both in species richness and diversity, was recorded during the rainy season $\left(\mathrm{DMg}=15.50, \mathrm{H}^{\prime}=1.15\right)$ at Pinzón reef. The dominant crustaceans were Balanus eburneus, Balanus improvisus, Eurypanopeus depressus and Petrolishtes armatus. Rev. Biol. Trop. 56 (Suppl. 1): 127-137. Epub 2008 May 30.
\end{abstract}

Key words: Oyster beds, diversity, richness, dominance, macrofauna, mollusks, crustaceans.

Las lagunas costeras son cuerpos acuáticos litorales que tienen en su mayoría comunicación permanente o efímera con el mar, se definen como el encuentro entre dos masas de agua de diferentes características (Contreras 1985). El estado de Tabasco cuenta con $183.86 \mathrm{Km}$. de cordón litoral y 29,800 Ha de superficies estuarinas. Posee dos lagunas costeras: El sistema lagunar Carmen-Machona y la laguna de Mecoacán, estas lagunas se encuentran habitadas por una gran variedad de peces, crustáceos y moluscos. Dentro de este último grupo se encuentra $C$. virginica que es un organismo gregario formador de bancos o arrecifes biogénicos, por lo que ha sido considerado como un ingeniero del ecosistema (Margalef 1968).

Los bancos ostrícolas también llamados camas, barras o arrecifes de ostión tienen un gran impacto en los ecosistemas costeros debido a los diferentes servicios que brinda, ayudan a mantener un ecosistema estable ya que se encargan de proveer hábitat y una fuente de alimento para varias especies, así como brindar protección a pequeños organismos tanto de las condiciones ambientales extremas así como de la depredación. Por otra parte las valvas de los ostiones brindan un sustrato duro para la fijación de especies como balanos, mejillones y otros organismos filtradores. (Kennedy et al. 1996). Hasta la fecha se han identificado más de 300 especies que dependen directa o indirectamente de los bancos ostrícolas (Wells 1961), dentro de estas se encuentran diversas especies de moluscos y crustáceos. El presente estudio se realizo en las lagunas costeras de Meacoacán, Carmen y Machona en el Estado 
de Tabasco, durante un periodo de tiempo que abarco d enero a diciembre de 2006, realizándose dos muestreos en cada una de las temporadas (secas, lluvias y nortes). El material colectado se transportaba al laboratorio de biología y cultivo de moluscos, donde la macrofauna bentónica (en particular crustáceos y moluscos) era separada, para posteriormente ser identificada. Con los datos obtenidos se calcularon índices de riqueza, diversidad y dominancia, con los cuales se realizaron Análisis de Varianza en cada una de las temporadas para identificar que bancos ostrícolas eran diferentes en cuanto al valor promedio de cada índice. Estudios similares se han realizado en las lagunas costeras de Tabasco, sin embargo son trabajos que evalúan crustáceos y moluscos por separado, no en conjunto como en este trabajo.

\section{MATERIALES Y MÉTODOS}

Área de estudio: El presente trabajo se realizó en el sistema lagunar de CarmenMachona que se localiza en el extremo oeste de la costa del estado de Tabasco. Se extiende entre los paralelos $18^{\circ} 15^{\prime}$ y $18^{\circ} 30^{\prime}$ de latitud $\mathrm{N}$ y los meridianos 9330' y 93․,35' de longitud W (Antolí \& García-Cubas 1985); así como en la laguna de Mecoacán, situada en el extremo oeste de la costa de Tabasco, cerca del poblado Puerto Ceiba. Se localiza entre las coordenadas $18^{\circ} 16^{\prime}$ y $18^{\circ} 26^{\prime}$ de latitud norte y $93^{\circ} 04^{\prime} y$ 93¹4'de longitud oeste. (García-Cubas et al. 1990). Se seleccionaron los siguientes bancos ostrícolas Puente de Ostión, Del Bajo y 87 para la Laguna de Mecoacán, Macayo, Largo y Pinzon para la Laguna Carmen y Los Ximénez y Chichal para la laguna Machona

Duración del estudio. El presente trabajo se llevo a cabo en un periodo de tiempo que abarcó desde marzo de 2006 a diciembre del mismo año. Se realizaron muestreos bimensuales, abarcando las temporadas de secas, lluvias y nortes. Durante la temporada de secas, disminuye el aporte de agua dulce a dichas lagunas, por lo que de manera general la salinidad aumenta, en la temporada de lluvias por el contrario hay un mayor aporte de agua dulce (debido a la precipitación característica de estas temporada) así como un aumento en la temperatura debido a que abarca verano, en la temporada de nortes, temperatura y salinidad disminuyen de manera importante.

Trabajo de campo. En cada banco seleccionado se tomó una muestra de cinco cuadrantes ( $n=5)$ de $20 \times 20 \mathrm{~cm}$. cada uno, lo que equivale a $0.2 \mathrm{~m}^{2}$ de cada banco. Se recolectaron todos los organismos presentes en cada cuadrante desprendiéndolos del sustrato y colocándolos en bolsas de plástico, debidamente etiquetadas. Los organismos se colocaron en neveras y se trasladaron al laboratorio de biología de moluscos del CINVESTAV-IPN, Unidad Mérida.

(Adicionalmente se registró en cada uno de los muestreos la temperatura, la salinidad y el oxígeno disuelto con la ayuda de un multianalizador YSY-85).

Trabajo de laboratorio. Se separaron los organismos del bentos asociado con la ayuda de un desconchador y un cuchillo, en el caso de balanos, mejillones y del ostión plano Isognomon alatus. Los cangrejos recolectados se colocaron en frascos de cristal con alcohol al $70 \%$ para su preservación y posterior identificación. Se identificó a las especies de crustáceos y moluscos asociados a los bancos ostrícolas mayores a $3 \mathrm{~mm}$. Se registró el número de individuos, peso húmedo y peso seco de cada una de las especies de moluscos identificadas con una balanza Sartorius de precisión 0.01 gr. En el caso de los crustáceos solo se obtuvo el peso húmedo.

Cálculo de la diversidad. Para calcular la diversidad $\alpha$ puntual se utilizaron métodos basados en la cuantificación del número de especies presentes (riqueza específica), así como métodos basados en la estructura de la comunidad (índices de dominancia y equidad), con el fin de que ambos parámetros sean complementarios en la descripción de la diversidad. De este modo se utilizó el Î́ndice de Margalef 
(Magurran 2001) para calcular la riqueza específica de especies, se determinó la abundancia mediante el Índice de Dominancia Simpson (Peet 1974, Magurran 2001) y la diversidad mediante el Î́ndice de Shannon-Wiener (Magurran 2001).

Para la determinación de estos índices se utilizo el software BioDiversity Professional Beta, Versión 2 . En el caso de los Índices de Margalef y Shannon-Wiener se utilizo el logaritmo en base 10, así como el inverso del Índice de diversidad de Simpson para calcular la dominancia de especies.

Análisis Estadístico. Se realizó un Análisis de Varianza en cada una de las temporadas para los diferentes bancos probando los supuestos de normalidad y homogeneidad de varianzas con las pruebas de Shapiro-Wilks modificado $\left(\mathrm{W}^{*}\right.$ y valor de p) y Levene respectivamente (valor de F y p.). Se realizó una prueba de comparaciones múltiples a posteriori (Test de Turkey) para identificar si existió diferencia en el valor promedio de los tres índices utilizados para medir diversidad entre los bancos ostrícolas, utilizándose los siguientes paquetes estadísticos: InfoStat/Profesional Versión 1.1 y STATISTICA Kernel release 5.5.

\section{RESULTADOS}

Para la macrofauna bentónica conformada por moluscos se identificaron en total 13 familias, 13 géneros y 15 especies de gasterópodos, así como 8 familias, 10 géneros y 11 especies de bivalvos. Durante la temporada de secas los valores del Índice de Margalef fueron normales y homocedásticos $\left(\mathrm{W}^{*}=0.83, \mathrm{p}=0.0823\right.$ y $\mathrm{F}=$ $2.7819 \mathrm{p}=0.0879$ ), existiendo diferencia significativa en el valor promedio de dicho índice entre los bancos $(\mathrm{F}=9,68, \mathrm{p} \leq 0.0001)$, siendo diferente de acuerdo al Test de Turkey el banco Puente de Ostión $(\mathrm{p} \leq 0.05)$, ya que fue el que registró la riqueza de especies promedio mas alta (Fig. 1). En lo que se refiere a la diversidad de especies y de acuerdo al análisis de varianza existió diferencia significativa en el valor promedio del Índice de Shannon-Wiener entre los seis bancos ostrícolas muestreados durante esta

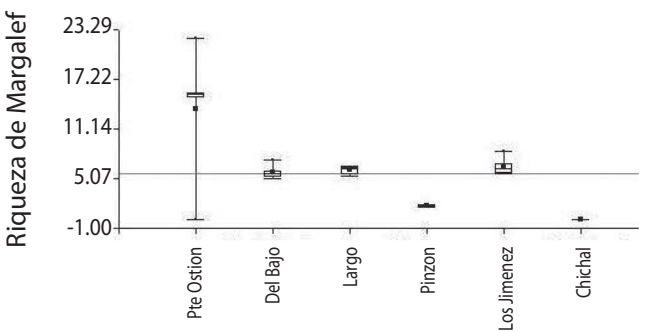

Banco A

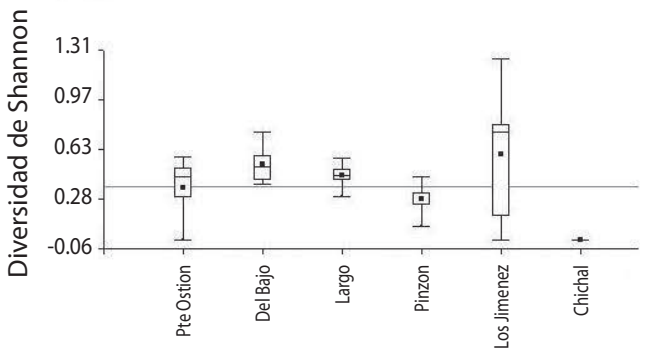

Banco B

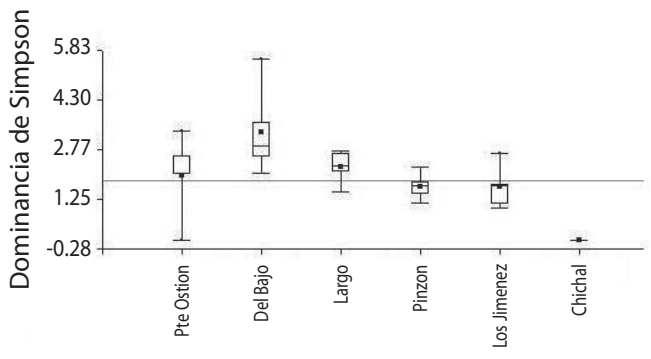

Banco C

Fig. 1. Comportamiento del valor promedio de los tres índices utilizados para medir la diversidad de moluscos durante la temporada de secas. (Pt. Ostión = Puente de Ostión). A) Índice de Riqueza de Margalef, B) Índice de Diversidad de Shannon-Wiener, C) Índice de Dominancia de Simpson. Las extensiones de las barras corresponden a la desviación estándar.

Fig. 1. Average percentage of the three indexes used to measure the mollusk diversity during the dry season. (Pt. Ostión = Puente de Ostión). A) Magalef's Index of Richness, B) Shannon-Wiener Index of Diversity, C) Simpson's Index of Dominance. The length of the bars indicates standard deviation.

temporada $\left(\mathrm{W}^{*}=0.91, \mathrm{p}=0.0943, \mathrm{~F}=2.1532\right.$, $\mathrm{p}=0.0698)(\mathrm{F}=3.75, \mathrm{p}=0.012)$, siendo los bancos Del Bajo, Largo y Los Jiménez diferentes al resto $(p \leq 0.05)$ ya que registraron una diversidad promedio de especies mayor a la de 
los demás bancos (Fig. 1). En cuanto al Índice de dominancia de Simpson, al igual que con los índices anteriores existió diferencia significativa en el valor promedio de los seis bancos ostrícolas $\left(\mathrm{W}^{*}=0.92, \mathrm{p}=0.1014, \mathrm{~F}=2.3035\right.$, $\mathrm{p}=0.0762)(\mathrm{F}=8.22, \mathrm{p}=0.0001)$. Los bancos Chichal, Los Jiménez y Pinzón fueron diferentes a los bancos los bancos Puente de Ostión y Largo debido a que presentaron los valores promedio más bajos de dicho índice (Fig. 1). El banco Del Bajo presentó los valores más altos de dominancia, en este banco las especies dominantes fueron Ischadium recurvum $\mathrm{y}$ Crepidula plana.

Durante la temporada de lluvias en lo que se refiere al Índice de Margalef existió diferencia significativa en el valor promedio de la riqueza entre los bancos ostrícolas $\left(\mathrm{W}^{*}=0.89\right.$, $\mathrm{p}=0.0723, \mathrm{~F}=2.2678, \mathrm{p}=0.0812)(\mathrm{F}=3.69$, $\mathrm{p}=0.0049$ ), siendo los bancos Pinzón, Macayo y Chichal diferentes de los demás bancos $(p \leq 0.05)$. En los dos primeros bancos los valores promedio de riqueza fueron los mas bajos, mientras que en el banco Chichal se registró la mayor riqueza promedio de especies (Fig. 2). En lo que respecta a la diversidad de ShannonWiener no existió diferencia significativa en el valor promedio de la diversidad de especies entre los bancos $\left(\mathrm{W}^{*}=0.95, \mathrm{p}=0.0821, \mathrm{~F}=\right.$ 1.9837, $\mathrm{p}=0.1021)(\mathrm{F}=1.27, \mathrm{p}=0.2972)$ por lo se podría considerar que la comunidad de moluscos de los bancos ostrícolas fue homogénea durante esta temporada, a diferencia de la temporada de secas (Fig. 2). En lo que se refiere a la dominancia de especies, al igual que con la diversidad no existió diferencia significativa en el valor promedio del Índice de dominancia de Simpson entre los bancos ostrícolas $\left(\mathrm{W}^{*}=0.90, \mathrm{p}=0.0799, \mathrm{~F}=2.6734\right.$, $\mathrm{p}=0.0684)(\mathrm{F}=1.47, \mathrm{p}=0.2145)$ (Fig. 2). Se obtuvo un valor promedio de dominancia de 2.55 bist/ind, pudiéndose considerar como las especies dominantes al mejillón I. recurvum, $I$. alatus y C. plana.

$\mathrm{Al}$ igual que en las dos temporadas anteriores, en la temporada de nortes existió diferencia significativa en el valor promedio del Índice de Riqueza de Margalef entre los ocho
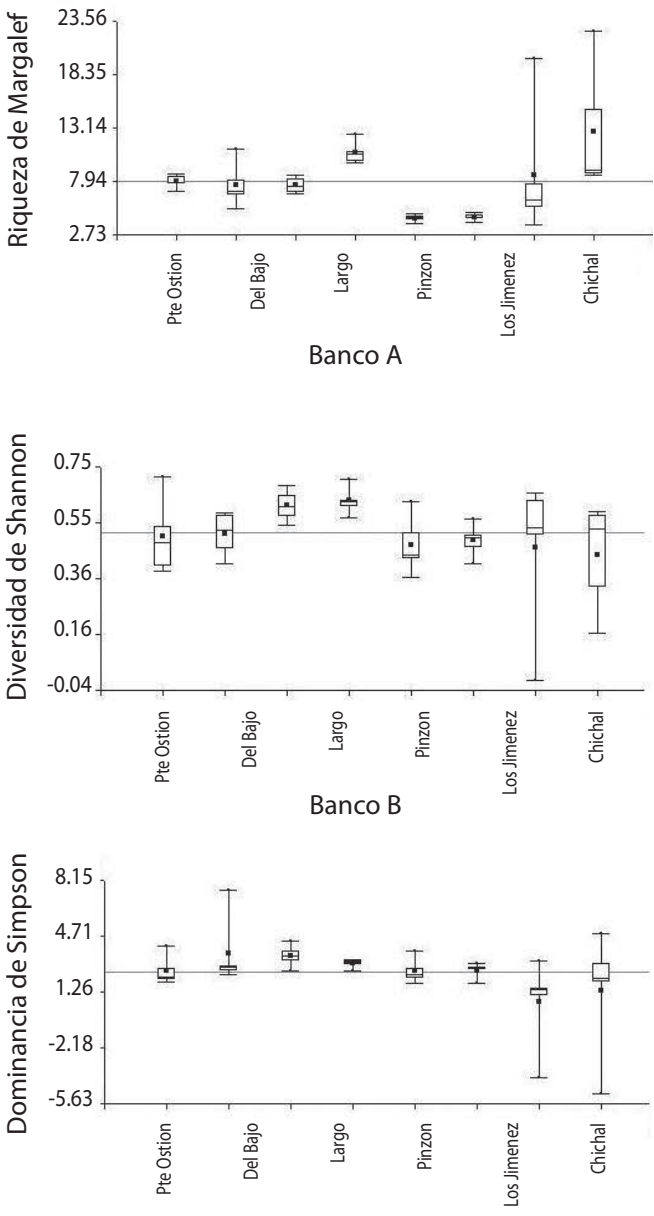

Banco C

Fig. 2. Comportamiento del valor promedio de los tres índices utilizados para medir la diversidad de moluscos durante la temporada de lluvias $($ Pt. Ostion = Puente de Ostión). A) Margalef, B) Shannon-Wiener, C) Simpson. Las extensiones de las barras corresponden a la desviación estándar.

Fig. 2. Average percentage of the three indexes used to measure the mollusk diversity during the rainy season. (Pt. Ostión = Puente de Ostión). A) Magalef, B) ShannonWiener, C) Simpson. The length of the bars indicates standard deviation.

bancos ostrícolas $\left(\mathrm{W}^{*}=0.93, \mathrm{p}=0.0967, \mathrm{~F}\right.$ $=2.0146, \mathrm{p}=0.0865) \quad(\mathrm{F}=7.07, \mathrm{p} \leq 0.0001)$, siendo diferentes los bancos Largo, Macayo, Los Jiménez y Chichal $(\mathrm{p} \leq 0.05)$, ya que registraron una riqueza promedio mayor a la de los 
demás bancos (Fig. 3). En cuanto al Índice de Shannon-Wiener se refiere, al igual que en la temporada de secas existió diferencia significativa en el promedio de la diversidad $\left(\mathrm{W}^{*}\right.$ $=0.94, \mathrm{p}=0.1302, \mathrm{~F}=1.8193, \mathrm{p}=0.1623$ ) $(\mathrm{p} \leq 0.05)$. Los bancos de la laguna Mecoacán registraron los valores más bajos de diversidad, mientras que los bancos de las lagunas Carmen y Machona tuvieron en comparación con los anteriores una diversidad promedio alta, a excepción del banco Pinzón, para el cual se calculó una diversidad ligeramente mas alta que en los bancos de Mecoacán (Fig. 3). Al igual que con los índices de diversidad y riqueza de especies existió diferencia significativa en el valor promedio de la dominancia por lo que al menos uno de los bancos ostrícolas fue diferente $\left(\mathrm{W}^{*}=\right.$ 0. 92, $\mathrm{p}=0.0983, \mathrm{~F}=2.2987, \mathrm{p}=0.0735)(\mathrm{F}=$ 12.07, $\mathrm{p} \leq 0.0001)$. Similar que con el índice de diversidad los bancos de la laguna de Mecoacán presentaron los valores de dominancia promedio mas bajos, y los bancos de las lagunas Carmen y Machona los mas altos (Fig. 3). Las especies dominantes durante esta temporada fueron $I$. recurvum, I. alatus y C. plana.

En lo que respecta a la macrofauna bentónica compuesta por crustáceos se identificaron un total de siete familias, 11 géneros y 12 especies. Durante la temporada de secas existió diferencia significativa en el valor promedio del Índice de Riqueza de Margalef entre los bancos ostrícolas $\left(\mathrm{W}^{*}=0.90, \mathrm{p}=0.9543, \mathrm{~F}\right.$ $=2.0233, \mathrm{p}=0.0843) \quad(\mathrm{F}=5.79, \mathrm{p}=0.0012)$, siendo el banco Puente de Ostión diferente de los demás bancos $(\mathrm{p} \leq 0.05)$ ya que presentó la riqueza promedio de especies mas alta (Fig. 4). A diferencia de la riqueza de especies no existió diferencia significativa en el valor promedio de diversidad de Shannon-Wiener entre los seis bancos analizados $\left(\mathrm{W}^{*}=0.95, \mathrm{p}=0.1127, \mathrm{~F}=\right.$ 1.9678, $\mathrm{p}=0.1003)(\mathrm{F}=2.77, \mathrm{p}=0.0409)$, por lo que la comunidad de crustáceos podría considerarse homogénea durante esta temporada (Fig. 4). En cuanto a la dominancia de especies, existió diferencia significativa en el promedio del Î́ndice de Simpson entre los bancos $\left(\mathrm{W}^{*}=\right.$ 0.91, $\mathrm{p}=0.0892, \mathrm{~F}=2.1622, \mathrm{p}=0.0786)(\mathrm{F}=$ 10.53, $\mathrm{p} \leq 0.0001)$. El banco ostrícola Chichal es

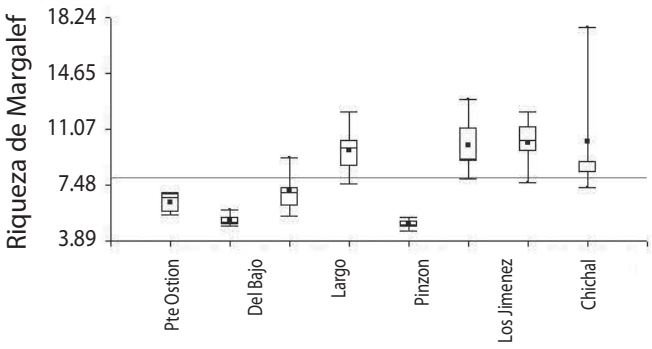

Banco A
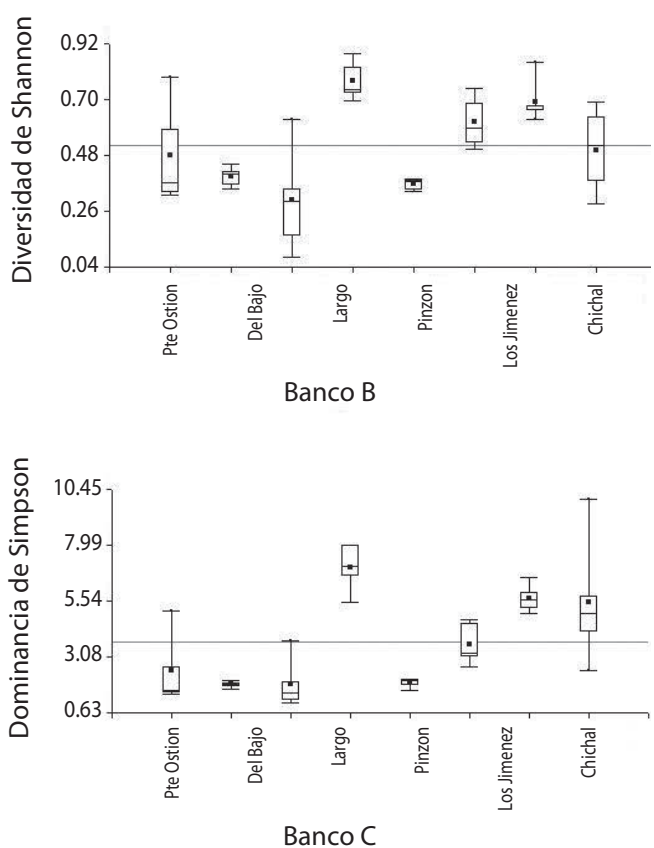

Fig. 3. Comportamiento del valor promedio de los tres índices utilizados para medir la diversidad de moluscos durante la temporada de nortes (Pt. Ostión =Puente de Ostión).A) Margalef, B) Shannon-Wiener, C) Simpson. Las extensiones de las barras corresponden a la desviación estándar.

Fig. 3. Average percentage of the three indexes used to measure the mollusk diversity during the "North wind" season. $($ Pt. Ostión = Puente de Ostión). A) Magalef, B) Shannon-Wiener, C) Simpson. The length of the bars indicates standard deviation.

diferente a los demás bancos en lo referente a dominancia $(\mathrm{p} \leq 0.05)$, ya que durante esta temporada no se registró ningún tipo de organismo asociado a este banco, siendo su dominancia la mas baja (Fig. 4). Las especies dominantes 


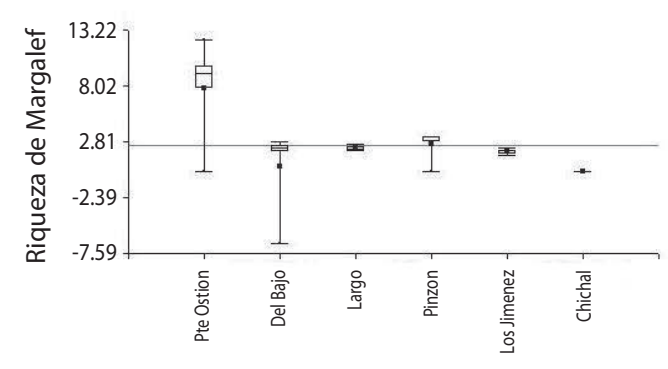

Banco A

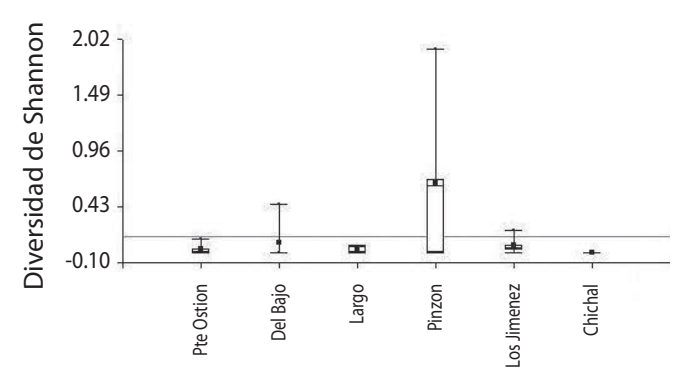

Banco B

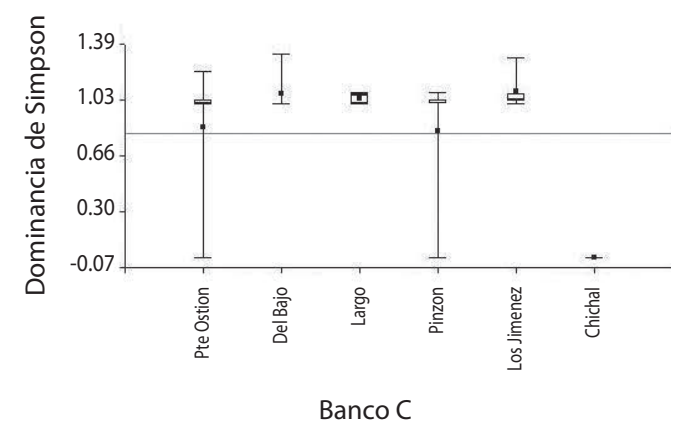

Fig. 4. Comportamiento del valor promedio de los tres índices utilizados para medir la diversidad de crustáceos durante la temporada de secas. (Pt. Ostión = Puente de Ostión). A) Margalef, B) Shannon-Wiener, C) Simpson. Las extensiones de las barras corresponden a la desviación estándar.

Fig. 4. Average percentage of the three indexes used to measure the crustacean diversity during the dry season. (Pt. Ostión = Puente de Ostión). A) Magalef, B) ShannonWiener, C) Simpson. The length of the bars indicates standard deviation.

para esta temporada fueron Balanus eburneus, Balanus improvisus y Eurypanopeus depressus.

En la temporada de lluvias se registra un cambio en el comportamiento de la riqueza de especies ya que a comparación de la temporada de secas no existió diferencia significativa en el valor promedio del Índice de Margalef entre los bancos $\left(\mathrm{W}^{*}=0.90, \mathrm{p}=0.0807, \mathrm{~F}=2.3462, \mathrm{p}=\right.$ 0.0604) $(\mathrm{F}=1.05, \mathrm{p}=0.4195)$. En comparación con la temporada de secas el banco Puente de Ostión registró una riqueza de especies baja durante la temporada de lluvias (Fig. 5). A diferencia de la temporada de secas existió diferencia significativa en el promedio del Índice de Diversidad de Shannon-Wiener entre los ocho bancos $\left(\mathrm{W}^{*}=0.92, \mathrm{p}=0.9861, \mathrm{~F}=1.8945\right.$, $\mathrm{p}=0.1034)(\mathrm{F}=11.36, \mathrm{p} \leq 0.0001)$, siendo los bancos Chichal y Pinzón diferentes $(\mathrm{p} \leq 0.05)$, al presentar la diversidad promedio de especies mas alta (Fig. 5). No existió diferencia significativa en el valor promedio del Índice de Dominancia de Simpson entre los ocho bancos ostrícolas $\left(\mathrm{W}^{*}=0.93, \mathrm{p}=0.1027, \mathrm{~F}=2.2984\right.$, $\mathrm{p}=0.0813)(\mathrm{F}=1.23$, gl: $7,39, \mathrm{p}=0.317)($ Fig. $5)$. Las especies dominantes al igual que en la temporada de secas fueron $B$. eburneus, $B$. improvistus y E. depressus.

En comparación con la temporada de lluvias en la temporada de nortes existió diferencia significativa en el promedio de la riqueza de especies $\left(\mathrm{W}^{*}=0.96, \mathrm{p}=0.5753, \mathrm{~F}=1.5319\right.$, $\mathrm{p}=0.1347)$ entre los ochos bancos ostrícolas analizados $(\mathrm{F}=11.9, \mathrm{p} \leq 0.0001)$. El banco Del Bajo fue el que presentó una mayor riqueza de especies, mientras que el banco Macayo fue el de menor riqueza (Fig. 6). Al igual que en las temporada de lluvias existió diferencia significativa en el valor promedio de diversidad entre los bancos ostrícolas $\left(\mathrm{W}^{*}=0.91, \mathrm{p}=0.0 .989, \mathrm{~F}\right.$ $=2.1748, \mathrm{p}=0.0879) \quad(\mathrm{F}=4.43, \mathrm{p}=0.0015)$. Así como con la riqueza de especies el banco Del Bajo registró la diversidad promedio más alta (Fig. 6). No existió diferencia significativa en el promedio de la dominancia $\left(\mathrm{W}^{*}=0.89\right.$, $\mathrm{p}$ $=0.0781, \mathrm{~F}=2.3102, \mathrm{p}=0.0683)(\mathrm{F}=2.34, \mathrm{p}=$ 0.0475) siendo Chichal y Macayo los bancos con mayor y menor dominancia promedio respectivamente (Fig. 6). Las especies dominantes en ésta temporada fueron $B$. eburneus, el porcelánido Petrolisthes armatus y el xánthido Hexapanopeus angustifrons. 


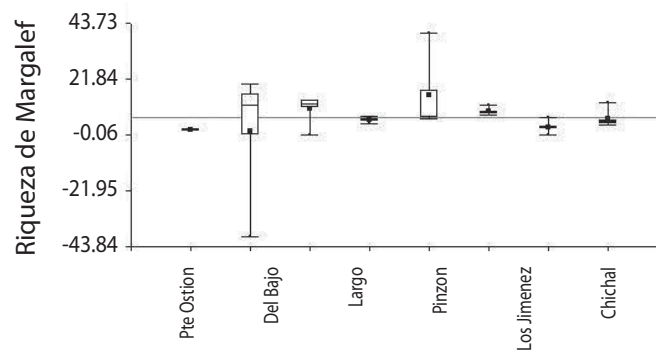

Banco A

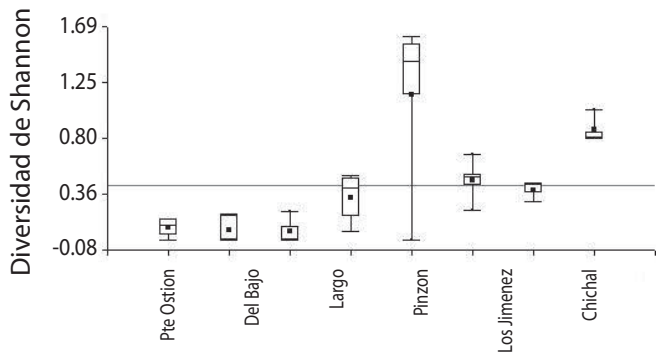

Banco B

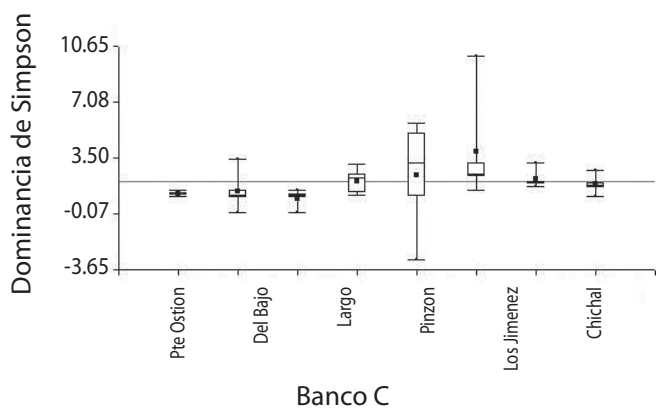

Fig. 5. Comportamiento del valor promedio de los tres índices utilizados para medir la diversidad de crustáceos durante la temporada de lluvias. (Pt. Ostión = Puente de Ostión). A) Margalef, B) Shannon-Wiener, C) Simpson. Las extensiones de las barras corresponden a la desviación estándar.

Fig. 5. Average percentage of the three indexes used to measure the crustacean diversity during the rainy season. (Pt. Ostión = Puente de Ostión). A) Magalef, B) ShannonWiener, C) Simpson. The length of the bars indicates standard deviation.

\section{DISCUSIÓN}

Existen varias definiciones de lo que es la diversidad alfa así como distintos métodos

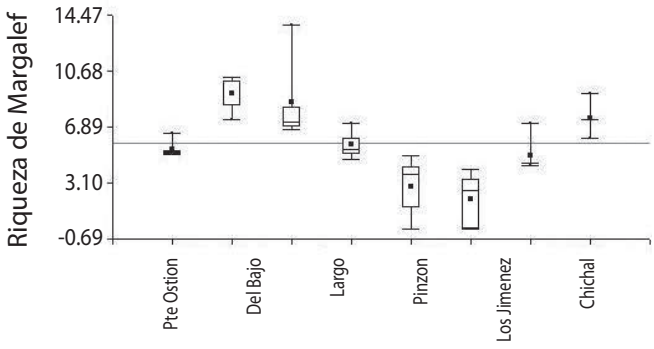

Banco A
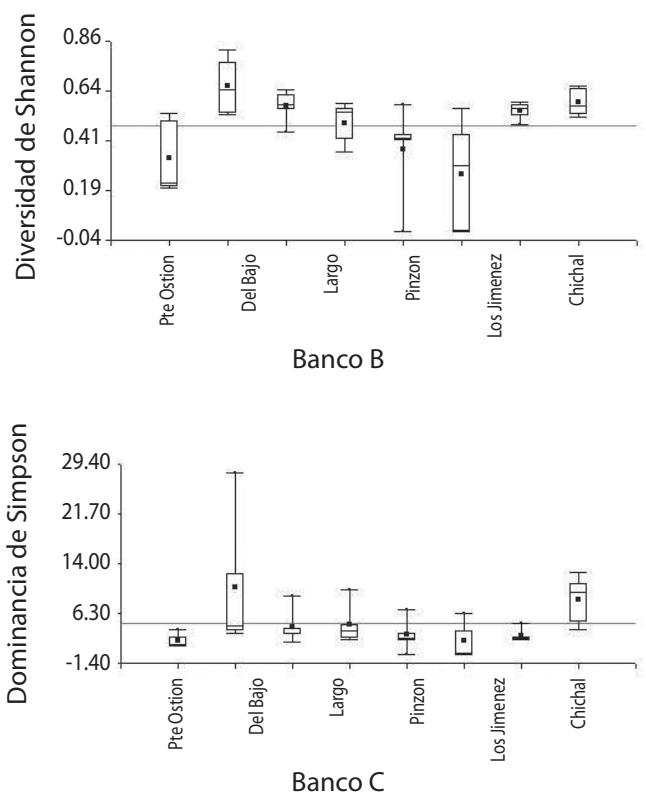

Fig. 6. Comportamiento del valor promedio de los tres índices utilizados para medir la diversidad de crustáceos durante la temporada de nortes. $($ Pt. Ostión = Puente de Ostión. A) Margalef, B) Shannon-Wiener, C) Simpson. Las extensiones de las barras corresponden a la desviación estándar.

Fig. 6. Average percentage of the three indexes used to measure the crustacean diversity during the "North wind" season. (Pt. Ostión = Puente de Ostión). A) Magalef, B) Shannon-Wiener, C) Simpson. The length of the bars indicates standard deviation.

e índices para evaluarla con el fin de tener una mejor representación de la misma durante este estudio se utilizaron métodos basados tanto en la riqueza como en la estructura de la 
comunidad. En lo que se refiere a los moluscos, en los bancos de la Laguna Mecoacán se identificaron un total de 13 especies distribuidos en 10 Familias de gasterópodos, así como siete especies distribuidos en seis Familias de bivalvos. Estos resultados son menores a los obtenidos por García Cubas et al. (1990) en la misma laguna, donde reportan 20 especies distribuidas en 11 Familias de gasterópodos y 22 especies agrupadas en 13 Familias de bivalvos. Esta diferencias pueden deberse a que el número de estaciones muestreadas en el estudio antes mencionado fue mayor al del presente trabajo, a pesar de esto el número de familias de gasterópodos es muy cercano al reportado por dichos autores. En lo que respecta a la macrofauna conformada por crustáceos, se registró un total de siete familias, 11 géneros y 12 especies. En la Laguna Mecoacán el banco Del Bajo registró el mayor número de especies de crustáceos con siete familias, 10 géneros y 11 especies, estos datos contrastan con lo obtenido por Domínguez et al. (2003) quienes reportan nueve Familias, 14 géneros y 19 especies de crustáceos distribuidos en toda la laguna. En el presente estudio los bancos ostrícolas de las lagunas Carmen y Machona presentaron un número similar de especies (siete Familias, 10 géneros, 10 especies, seis Familias, ocho géneros y nueve especies, respectivamente).

Temporada de Secas. Durante esta temporada la riqueza de especies más alta se registró en el banco Puente de Ostión, ubicado en una zona de baja salinidad (23 en promedio). Sin embargo los valores de diversidad mas altos se registraron en el banco Los Jiménez, situado en una zona de alta salinidad (32 en promedio), lo anterior coincide con lo descrito con Parker (1975) quien menciona que los bancos ostrícolas situados en zonas de baja salinidad generalmente presentan una baja diversidad de especies, con la presencia de pocas poblaciones de elevada biomasa, mientras que aquellos bancos que se encuentran en zonas con altas salinidades presentan valores intermedios de diversidad, así como poblaciones de niveles intermedios. Esto también se confirmó con los valores resultantes del Índice de Dominancia de Simpson, en donde el banco Del Bajo ubicado en una zona de baja salinidad fue el que presentó los valores más altos de dominancia, en este banco las especies dominantes fueron Ischadium recurvum y Crepidula plana. García Cubas et al. (1990) mencionan que I. recurvum fue la especie viva más abundante que recolectó en su estudio, estando esta restringida a una sola estación frente a la Punta Tilapa (en la Laguna de Mecoacán), esto contrasta parcialmente con lo obtenido durante el presente trabajo, ya que si bien esta especie fue abundante, no se encontró restringida solo a ésta zona en la Laguna de Mecoacán.

Durante esta temporada la comunidad o ensamble de crustáceos fue homogéneo ya que los valores de diversidad promedio no fueron diferentes, es decir las especies que las especies encontradas en los bancos ostrícolas fueron similares. Los valores del índice de dominancia de Simpson en general fueron bajos (0.81 en promedio), las especies dominantes fueron Balanus eburneus, Balanus improvisus y Eurypanopeus depressus. Estos resultados son similares a los obtenidos por Meyer y Towsend (2000) quienes observaron la utilización de bancos ostrícolas artificiales por diferentes grupos taxonómicos, dividiéndolos en fauna sésil y fauna móvil dentro de la fauna sésil, la especie abundante fue $B$. eburneus así como dos especies de moluscos mientras que las especies mas abundantes de fauna móvil fueron los cangrejos de la Familia Xanthidae Panopeus herbstii y E. depressus.

Temporada de lluvias. Los bancos ostrícolas Largo y 87 presentan durante esta temporada los valores más altos de los tres índices utilizados para medir la diversidad alfa. En cuanto a la riqueza y diversidad de especies se obtuvo un valor promedio de 7.1 y 0.51 bits/ ind. respectivamente, siendo ligeramente mas altos a los obtenidos durante la temporada de secas (5.87 y 0.35 bits/ind). Esto podría ser explicado por la adición de especies tales como $N$. vivex, O. impressa, $M$. leucophaeta y M. corona. No obstante esto contrasta con lo 
obtenido por García-Cubas et al. (1992) en el sistema Lagunar Chica-Grande, en el estado de Veracruz, quienes registraron valores de riqueza de especies de 0.61 a 2.08 bits/ind. obteniéndose de manera general una mayor riqueza de especies de moluscos en los bancos ostrícolas de las tres lagunas de Tabasco, sin embargo en la diversidad de especies del presente estudio fue menor a la de dicho sistema lagunar (0.33 a 3.25 bits/ind.). En lo que respecta a la dominancia de especies se obtuvo un valor promedio de 2.55 bist/ind, pudiéndose considerar como las especies dominantes al mejillón I. recurvum, al ostión plano I. alatus así como a C. plana, la cual es una especie que se reproduce durante todo el año, alcanzando sus picos de reproducción durante esta temporada (Griffin 2001). Lo anterior explica la dominancia de ésta especie en la mayoría de los bancos ostrícolas estudiados en la misma época.

En lo que se refiere a la macrofauna conformada por crustáceos, no existió diferencia en el valor promedio de la riqueza de especies, por lo que se asume que en los ocho bancos se presentó un número similar de especies. Sin embargo, en lo que respecta a la diversidad los bancos de las lagunas Carmen y Machona fueron mas diversos, con un valor promedio de diversidad de (0.43 bits/ind.), este resultado es similar al obtenido por Tolley et al. (2005) en bancos ostrícolas del estero Caloosahatchee, Florida, donde se reporta un valor promedio del Índice de Shannon durante la temporada de lluvias de (0.427 bits/ind.), sin embargo en este mismo estudio se reportan valores de diversidad promedio menores a los obtenidos en los bancos Pinzón y Chichal en las lagunas Carmen y Machona (1.15 y 0.87 bits/ind., respectivamente). Las especies dominantes al igual que en la temporada de secas fueron $B$. eburneus, B. improvistus y E. depressus. Esto puede deberse a que organismos como los balanos desempeñan diversas funciones dentro de la comunidad de los bancos ostrícolas; así por ejemplo se sabe que $B$. eburneus juega un rol tanto como comensal (ya que generalmente se fija a las conchas de los ostiones sin causarles daño), como competidor ya que al ser organismos son filtradores compiten por alimento (Kilgen \& Dugas 1989).

Temporada de nortes. El banco ostrícola Largo (Laguna Carmen) presentó para esta temporada los valores más altos de los tres índices, riqueza, diversidad y dominancia de especies (13, 0.90 y 7.99 bits/ind respectivamente). Los datos de diversidad son menores a lo obtenido por Antolí y García-Cubas (1985), quienes realizaron un estudio sobre la sistemática de los moluscos del sistema lagunar CarmenMachona, reportando que la diversidad de moluscos mas baja del sistema se registró en la Laguna Carmen (1.76 bits/ind.), siendo mayor la diversidad en la Laguna Machona (2.10 bits/ ind.). Como se mencionó anteriormente la diferencia entre estos resultados puede deberse a que el presente estudio se realizó en zonas donde se ubican bancos ostrícolas en cada una de de las lagunas, mientras que el realizado por Antolí y García-Cubas (1985) abarco todo el sistema lagunar. En el presente estudio las especies dominantes durante esta temporada fueron I. recurvum, I. alatus y C. plana. Zimmerman et al. (1989) reportó densidades promedio de C. plana de 1 Ind./78.5 $\mathrm{cm}^{2}$., en bancos de ostrícolas de West Bay, Texas durante la temporada de invierno (algunos de invierno contemplan parte de la temporada de nortes en nuestro estudio), siendo dominante al igual que en el presente trabajo, y ocupando el tercer lugar en abundancia dentro de los moluscos reportados por éste autor. Rodney y Paynter (2006) menciona que de la fauna asociada a bancos ostrícolas restaurados y sin restaurar en Chesapeake Bay, Maryland los organismos que se fijan al ostión (llamados "fouling”) fueron más abundantes en los bancos restaurados, siendo el mejillón I. recurvum la especie dominante.

Durante esta temporada existió diferencia en los valores promedio tanto de riqueza como de diversidad de especies de crustáceos, siendo los bancos Puente de Ostión, Pinzón y Macayo en los que se registraró los valores más bajos de ambos índices. Las especies dominantes en ésta temporada fueron $B$. eburneus, el por- 
celánido Petrolisthes armatus y el xánthido Hexapanopeus angustifrons. Este comportamiento es similar al observado por Zimmerman et al. (1989) quienes mencionan que los crustáceos decápodos dominantes en los bancos ostrícolas durante el invierno son los palemónidos así como los cangrejos de las familias Xanthidae y Porcellanidae.

\section{AGRADECIMIENTOS}

Al CONACYT por el apoyo proporcionado para llevar a cabo mis estudios de maestría con especialidad en Biología Marina, en el CINVESTAV, IPN, Unidad Mérida. A Jorge Montero por su asesoría estadística. A la AMLC por la ayuda otorgada para asistir a su 33ra reunión en St. Thomas, U. S. V. I.

\section{RESUMEN}

El ostión Crassostrea virginica es nativo de la costa norteamericana del Atlántico. En México se distribuye desde Laguna Madre, Tamaulipas, hasta Celestún, Yucatán. Habita en áreas estuarinas y esporádicamente en aguas marinas. Este ostión tiene importancia comercial, social y ecológica. A nivel ecológico los bancos que forma $C$. virginica proveen hábitat para invertebrados, entre ellos moluscos y crustáceos, que viven en asociación con este. En el presente trabajo se realizaron muestreos durante las temporadas de secas, lluvias y nortes, en ocho bancos ostrícolas de las lagunas de Mecoacán, Carmen y Machona, en Tabasco, México. Los bancos ostrícolas seleccionados fueron Puente de Ostión, Del Bajo, 87, Largo, Pinzon, Macayo, Los Jiménez y Chichal. En cada banco se muestreó por medio de cinco cuadrantes de 20 x 20 cm (equivalentes a $0.02 \mathrm{~m}^{2}$ ). Para cada cuadrante se registraron las especies de moluscos y crustáceos asociados a los bancos ostrícolas (número, peso húmedo total y peso seco). Se calculó la diversidad $\alpha$ puntual mediante los índices de diversidad de Shannon-Wiener, dominancia de Simpson y Margalef. Se realizó un Análisis de Varianza para evaluar si existía diferencia en el promedio de cada uno de los índices en cada una de las temporadas. En lo que se refiere a la riqueza de especies de moluscos, se encontraron en total 13 familias, 13 géneros y 15 especies de gasterópodos, así como 8 familias, 10 géneros y 11 especies de bivalvos. En lo que respecta a los crustáceos se registró un total de siete familias, 11 géneros y 12 especies. En el caso de los moluscos el valor promedio de la riqueza de especies más alto se registró durante la temporada de secas en el banco Puente de Ostión $\left(\mathrm{D}_{\mathrm{Mg}}=13.6\right)$, mientras que en el caso de la diversidad el valor promedio mas alto se presentó en la temporada de nortes, en el banco Largo $\left(\mathrm{H}^{\prime}=0.77\right.$ bits/ ind.), las especies de moluscos que fueron dominantes durante las tres temporadas fueron Ischadium recurvum, Isognomon alatus y Crepidula plana. En lo que se refiere a los crustáceos, el valor promedio mas alto tanto de la riqueza como de la diversidad de especies se registraron en la temporada de lluvias ( $\mathrm{D}_{\mathrm{Mg}}=15.50, \mathrm{H}^{\prime}=1.15$ bits/ind. $)$ en el banco Pinzón. Las especies de crustáceos dominantes fueron Balanus eburneus, Blanus improvisus, Eurypanopeus depressus y Petrolishtes armatus. La diversidad de especies de crustáceos y moluscos varió durante las temporadas de secas, lluvias y nortes. De manera general el número de especies de moluscos gasterópodos fue mayor al de bivalvos; sin embargo, los bivalvos presentaron una mayor abundancia durante las tres temporadas. Los crustáceos estuvieron representados principalmente por cangrejos de las familias Xanthidae y Porcellanidae, así como por los cirrípedos de la familia Balanidae.

Palabras clave: bancos de ostrícolas, diversidad, riqueza, dominancia, macrofauna, moluscos, crustáceos.

\section{REFERENCIAS}

Antolí, A. \& A. García-Cubas. 1985. Sistemática y ecología de moluscos en las lagunas costeras Carmen y Machona, Tabasco, México. Anales Inst. Cien. Mar Limnol. UNAM 12: 145-198.

Contreras, F. 1985. Las lagunas costeras mexicanas. Centro de Ecodesarrollo, Secretaria de Pesca, México DF. 253 pp.

Domínguez, J.C., A.J. Sánchez, R. Florido \& E. Barba. 2003. Distribución de macrocrustáceos en Laguna Mecoacán, al sur del Golfo de México. Hidrobiologia 13: 127-136.

García-Cubas, A, F. Escobar, L.V. González \& M. Reguero. 1990. Moluscos de la Laguna Mecoacán, Tabasco, México: sistemática y ecología. Anales Inst. Cienc. Mar Limnol. UNAM 17: 1-30

García-Cubas, A, M. Reguero \& R. Elizarrarás. 1992. Moluscos del sistema Lagunar Chica-Grande, Veracruz, México: sistemática y ecología. Anales Inst. Cienc. Mar Limnol. UNAM 19: 71-121.

Kennedy, V.S., R.I.E. Newell \& A.F. Eble. 1996. The Eastern Oyster, Crassostrea virginica. Maryland Sea Grant, UM-SG-TS-96-01: 734 p.

Kilgen, R.H. \& R.J. Dugas. 1989. The Ecology of Oyster Reefs of The Northern Gulf of Mexico: An Open File Report. NWRC 89-02: 113 p.

Magurran, A.E. 2001. Ecological diversity and its measurement. In: C.E. Moreno (ed.). Métodos para medir 
la biodiversidad. Vol. 1. M\&T-Manuales y Tesis, Zaragoza, España.

Margalef, R. 1968. Perspectives in ecological theory. Ann. Rev. Ecol. Evol. Syst. 36: 643-89.

Meyer, D.L. \& E.C. Townsend. 2000. Faunal utilization of created intertidal eastern oyster (Crassostrea virginica) reefs in the southeastern United States. Estuaries 23: 34-45.

Parker, R.H. 1975. The study of benthic communities a model and review. In: R.H. Kilgen \& R.J. Dugas. 1989. The Ecology of Oyster Reefs of The Northern Gulf Of Mexico: An Open File Report. NWRC 89-02: 113 p.

Peet, R.K. 1974. The measurement of species diversity. Annual Review of Ecology and Systematics In: Moreno, C.E. 2001. Métodos para medir la biodiversidad. M\&T-Manuales y Tesis SEA, Vol. 1. Zaragoza, España. 84 p.

Rodney, W.S. \& K.T. Paynter. 2006. Comparisons of macrofaunal assemblages on restored and non-restored oyster reefs in mesohaline regions of Chesapeake Bay in Maryland. J. Exp. Mar. Biol. Ecol. 335: 39-51.

Tolley, G.S., A.K. Volety \& M. Savarese. 2005. Influence of salinity on the habitat use of oyster reefs in three Southwest Florida estuaries. J. Shellfish Res. 24: 127-138.

Wells, H.W. 1961. The fauna of oyster beds, with special reference to the salinity factor In: Tolley, G.S, Volety, A.K and Savarese, M. 2005. Influence of salinity on the habitat use of oyster reefs in three Southwest Florida estuaries. J. Shellfish Res. 24: 127-138.

Zimmerman, R.J., T. Minello, T.J. Baumer \& M. Castiglione. 1989. Oyster reef as habitat for estuarine macrofauna. NOAA Tech. Memo. NMFS-SEFC-249: 16 p.

\section{REFERENCIAS DE INTERNET}

Griffin, T. 2001. Florida Institute of Technology. Smithsonian Marine Station at Fort Piece. irl_webmaster@serc.si.edu. 
